\title{
The spatial representation of numbers: evidence from neglect and pseudoneglect
}

\author{
Carlo Umiltà · Konstantinos Priftis · Marco Zorzi
}

Received: 23 June 2008 / Accepted: 14 October 2008 / Published online: 5 November 2008

(C) Springer-Verlag 2008

\begin{abstract}
The aim of the present paper is to provide an overview of the evidence that links spatial representation with representation of number magnitude. This aim is achieved by reviewing the literature concerning the number interval bisection task in patients with left hemispatial neglect and in healthy participants (pseudoneglect). Phenomena like the Spatial Numerical Association of Response Codes (SNARC) effect and the shifts of covert spatial attention caused by number processing are thought to support the notion that number magnitude is represented along a spatially organized mental number line. However, the evidence provided by chronometric studies is not univocal and is open to alternative, non-spatial interpretations. In contrast, neuropsychological studies have offered convincing evidence that humans indeed represent numbers on a mental number line oriented from left to right. Neglect patients systematically misplace the midpoint of a numerical interval they are asked to bisect (e.g., they say that $\langle 5\rangle$ is halfway between $\langle 2\rangle$ and $\langle 6\rangle$ ) and their mistakes closely resemble the typical pattern found in bisection of true visual lines. The presence of dissociations between impaired explicit knowledge and spared implicit knowledge supports the notion that neglect produces a deficit in accessing an intact mental number line, rather than a distortion in the representation of that line. Other results show that the existence of a strong spatial connotation constitutes
\end{abstract}

C. Umiltà $(\bowtie) \cdot$ K. Priftis · M. Zorzi

Dipartimento di Psicologia Generale,

Università di Padova, Padua, Italy

e-mail: carlo.umilta@unipd.it

K. Priftis

IRCCS San Camillo, Lido-Venezia, Italy a specific property of number representations rather than a general characteristic of all ordered sequences.

Keywords Mental number line - Numerical cognition · Spatial representation $\cdot$ Neglect $\cdot$ Pseudoneglect .

Number bisection

\section{The spatial coding of numerical magnitude}

The most influential model of numerical cognition, the Triple Code Model (e.g., Dehaene 1992; Dehaene et al. 2003) assumes that numbers are mentally represented in three different ways. One is a visual, Arabic code, in which numbers are represented as strings of visual forms (e.g., $\langle 21453\rangle)$. The second is a verbal code, representing numbers as sequences of number-words syntactically organized (e.g., 〈two-thousands four-hundred and fifty-two〉). The third code is a representation of numerical magnitude that is assumed to be spatial in nature: Numbers (perhaps singleand double-digit ones only) would be represented as local activations (points or regions) along a mental number line (MNL; e.g., Dehaene 2003). The spatial code would be at the core of number meaning because the semantic value of a number, its magnitude, would be conveyed by its position on the MNL. In people who read from left to right, the MNL is spatially oriented from left to right, with smallmagnitude numbers represented on the left-hand side of the line and numbers of larger magnitude represented on the right-hand side.

Even though the intuition of an MNL, in which numerical magnitude is spatially represented, dates back to Galton (1880), empirical evidence came much later. The finding that it is easier and quicker to select the larger of two numbers when they are numerically distant than when they are 
closer (for example, $\langle 8\rangle$ and $\langle 3\rangle$ are compared faster than $\langle 8\rangle$ and $\langle 6\rangle$ ), referred to as the distance effect (Moyer and Landauer 1967), was thought to reflect a representation of numbers as points on a continuous, analogue MNL (Restle 1970). Thus, the time to decide which is the larger of two numbers would be a function of the distance between them on the MNL. The farther apart two numbers are, the easier it is to compare them. Although this view is endorsed by current theories of number representation (e.g., Dehaene 2003; Gallistel and Gelman 2000), the distance effect speaks of an analogical representation but it does not necessarily entail a spatial representation of numbers. Indeed, a distance effect is found whenever the comparison task involves physical quantities (e.g., Holyoak 1977; Kosslyn et al. 1977) or even the abstract ordering of elements in non-numerical sequences (e.g., Woocher et al. 1978).

A phenomenon that has long been thought to provide direct evidence of the link between the domain of numerical magnitude and the domain of space is the SNARC effect (Dehaene et al. 1993). Even though the SNARC effect was first obtained in a numerical comparison task with two-digit Arabic numbers (Dehaene et al. 1990), it is now typically investigated with a parity (odd/even) judgment task. When performing parity judgments to centrally presented single numbers, participants respond faster with the effector that operates on the left side of space to relatively small numbers and with the effector that operates on the right side of space to relatively large numbers. In other words, even if number magnitude is task-irrelevant and the number is presented at fixation, an interaction is obtained between side of the response and magnitude of the number. The classic explanation of the SNARC effect is based on the notion of an analogue, left-to-right oriented MNL, with relatively small numbers on the left and relatively large numbers on the right (Dehaene et al. 1993). The SNARC effect arises because of a spatial correspondence, or lack of correspondence (i.e., corresponding vs. non-corresponding trials), between position of the number on the MNL and position of the response. It was subsequently shown that the SNARC effect can be obtained with various effectors, such as hands, fingers of the same hand (Priftis et al. 2006), feet (Schwarz and Müller 2006), and saccades (Fischer et al. 2004; Schwarz and Keus 2004).

It has been argued (Gevers et al. 2006b; Keus and Schwarz 2005) that the SNARC effect arises during selection of the spatial (left vs. right) response. Indeed, the SNARC effect correlates better with response-locked than stimulus-locked scalp potentials (Gevers et al. 2006a; Keus et al. 2005) and disappears, in parity judgment tasks, when left and right responses are replaced by verbal, non-spatial responses (Keus and Schwarz 2005).

None of the many studies on the SNARC effect, however, provided direct, unequivocal support for a spatial representation of numbers (see Proctor and Cho 2006; Santens and Gevers 2008). Indeed, Gevers et al. (2006b) offered a computational account of the SNARC effect that does not assume a spatial organization of the mental number line: "a specific number is not coded as left or right but $(\ldots)$ is coded as either small or large, which in turn activates left or right responses" (p. 41).

Further evidence of the relation between space and numerical magnitude comes from studies of spatial attention. Fischer et al. (2003) showed that the mere perception of a number causes a shift in covert attention to the left or right side of space, depending on number magnitude. Participants were required to detect as fast as possible a peripheral target that was preceded by presentation of an irrelevant digit at fixation. Results indicated that left targets were detected faster when preceded by small digits $(\langle 1\rangle$ or $\langle 2\rangle)$, whereas detection of right targets was faster when preceded by larger digits $(\langle 8\rangle$ or $\langle 9\rangle)$. Galfano et al. (2006; also see Ristic et al. 2006) confirmed the effect of number magnitude on spatial attention. Their results, however, were in contrast with the notion that orienting caused by number magnitude is automatic in nature (also see Bonato et al. 2008b).

In a study by Casarotti et al. (2007), the relation between numbers and spatial attention was examined using temporal order judgments (TOJ). Results confirmed that, as a consequence of numerical processing, spatial attention shifted automatically to the left or right side of space. That, in turn, affected the speed with which sensory information was transmitted in the visual system. Given equal onset time, left-side stimuli were perceived to occur before right-side stimuli when a small number was presented at fixation, whereas right-side stimuli were perceived to occur before left-side stimuli when a larger number was presented. Importantly, the effect was contingent upon the explicit processing of the number as a secondary task demand, which casts further doubts on the automatic nature of the shift of spatial attention.

Although response selection was irrelevant in all tasks involving shifts of spatial attention, minor modifications of Gevers et al.'s (2006b) model could still account for these results, with an automatic categorization of the central number as small or large, and a consequent activation of left or right spatial codes. If numbers are represented spatially, the finding of an influence of number processing upon spatial orienting (as in Casarotti et al. 2007; Fischer et al. 2003) should be complemented by the finding that spatial processing influences numerical processing. This issue was addressed in a recent study of Stoianov et al. (2008). Stoianov et al. noted that spatial coding of visual stimuli is fast and automatic (Lu and Proctor 1995; Zorzi and Umiltà 1995), whereas spatial coding of numbers on the MNL line is relatively slow, requiring both perceptual 
processing (digit recognition) and semantic processing (activation of the MNL). Accordingly, they hypothesized that visual-spatial priming in non-spatial, verbal tasks, which exclude response-selection bias, would depend on the concurrent activation of perceptual and numerical spatial representations. Indeed, in both number comparison and parity judgment, they found that a spatial cue primes a target number more if the cue follows (backward priming) than if it precedes (forward priming) the target.

In summary, although the behavioral studies on healthy participants support the hypothesis that the representation of numbers is spatial in nature, the available evidence (perhaps with the exception of Stoianov et al. 2008) does not appear to be conclusive. In contrast, neuropsychological studies have offered strong and compelling evidence, the strongest and most compelling evidence to date in fact, that humans indeed represent numbers on a MNL oriented from left to right. These studies are the main focus of the present review article.

\section{Neglect and the mental number line}

Zorzi et al. (2002) investigated the issue of the spatial nature of the MNL from a different perspective, that is by exploring the way numbers are represented in patients with left (hemi)spatial neglect. Patients with left neglect, following a right parietal lesion, fail to report, orient to, or verbally describe stimuli in the contralesional left (hemi)space (for reviews, see, e.g., Halligan et al. 2003; Heilman et al. 1979). For instance, when they have to mark the midpoint of a linear segment positioned in front of them, they systematically displace the midpoint to a subjective midpoint located on the right of the true midpoint, as if they ignored the leftmost part of the segment. As was reported by Halligan and Marshall (1988; also see Marshall and Halligan 1989), the rightward displacement is directly proportional to the length of the segment: The longer the segment, the greater the rightward displacement. However, a paradoxical phenomenon is observed for very short segments, which consists in a leftward displacement of the midpoint (i.e., the so-called crossover effect). Similar effects of left neglect on line bisection were reported in studies that required the bisection of imagined linear segments (Bisiach et al. 1994; Chokron et al. 1997). This is in accord with the well-known fact that neglect is not confined to stimuli that are actually present in the environment, but extends also to mental images that are actively produced by the observer (e.g., Bisiach and Luzzatti 1978).

Zorzi et al. (2002) reasoned that, if the MNL is more than a mere metaphor but rather refers to a representation that is truly spatial in nature, neglect patients would show the same form of shift in bisecting a numerical interval as they show in the line bisection task. In their study, participants were presented with two numbers (e.g., $\langle 3\rangle$ and $\langle 9\rangle$ ), which defined the to-be-bisected numerical interval. The task consisted in telling aloud the number that occupied the middle position in the given interval (e.g., $\langle 6\rangle$ ). Neglect patients systematically misplaced the midpoint of the numerical interval (e.g., responding that $\langle 5\rangle$ is halfway between $\langle 2\rangle$ and $\langle 6\rangle)$ and their mistakes closely resembled the typical pattern found in bisection of true visual lines, including the modulating effect of line length and the crossover effect with very short numerical intervals. ${ }^{1}$

This new form of representational neglect was considered to be evidence that the MNL is more than a metaphor and that its spatial nature renders it functionally isomorphic to visual lines. Moreover, the demonstration of left-right orientation was indeed stronger and more direct than that provided by the chronometric data from the SNARC task.

The original findings of Zorzi et al. (2002) were replicated and extended in a number of subsequent studies, which used number interval bisection and similar tasks (Cappelletti et al. 2007; Doricchi et al. 2005; Hoeckner et al. 2008; Loftus et al. 2008; Priftis et al. 2006; Zamarian et al. 2007; Zorzi et al. 2006). The most important findings will be reviewed in the following sections.

Number line versus physical lines

Zorzi et al. (2002) claimed that the mental number line is isomorphic to visual lines. That is, the MNL has properties that are analogue to those of visual lines. Nonetheless, the MNL is a representation in the imaginal space, whereas visual lines are representations in the perceptual space. Because the two spaces (imaginal vs. perceptual) can be doubly dissociated in neglect patients (Anderson 1993; Guariglia et al. 1993), it is not surprising that number interval bisection can be doubly dissociated from visual line bisection. Indeed, Zorzi et al. (2004) reported a double dissociation between number interval bisection and visual line bisection in two single cases. That issue was later investigated also by Doricchi et al. (2005), who showed the double dissociation between visual line bisection and number interval bisection in a group study on neglect patients. Doricchi et al. maintained that their results disproved Zorzi

\footnotetext{
${ }^{1}$ Zorzi et al.'s (2002) neglect patients were unimpaired in arithmetical tasks, which, at first sight, seems to contradict the notion that quantity is represented spatially. However, Priftis et al. (2006) have subsequently shown that in neglect patients, spatial representations of numbers are intact and only explicit access to those spatial representations is impaired. In addition, it should be kept in mind that Zorzi et al.'s neglect patients were perfectly able to bisect the number interval by applying an algorithm, that is, through the use of Dehaene's (1992) verbal code (see above). However, they were strictly asked not to do so.
} 
et al.'s (2002) hypothesis of a representational isomorphism between number line and physical lines. Moreover, they claimed that the deficit in number interval bisection can be attributed to a spatial working memory disorder that affects "short term retention of contralateral positions" following damage to right prefrontal cortex.

Setting the anatomical issue aside (note that many neglect patients included in later studies showed a number interval bisection bias even though there was no hint of prefrontal damage; e.g., Priftis et al. 2006; Zorzi et al. 2006), it is worth pointing out that Zorzi et al. (2002) did not claim that the number line is represented as a visual line. Functional isomorphism implies that the representation of the number line is based on the same spatial metric that underlies the representation of visual lines but it does not require any shared neural mechanism. Moreover, Doricchi et al.'s hypothesis that the critical deficit is one involving spatial working memory falls short in explaining the effect of neglect on much simpler tasks, such as number comparison (Vuilleumier et al. 2004) or detection of a target number (Priftis et al. 2008) (see below), which are unlikely to involve spatial working memory.

As noted in the previous section, number processing has been found to influence spatial processing in chronometric studies. Bonato et al. (2008a) showed that this influence is also observed in the line bisection performance of neglect patients. Indeed, they observed that the size of the bisection bias was modulated by numbers presented as irrelevant flankers on both sides of the visual line. The typical rightward bias increased when the flankers were two identical digits $\langle 9\rangle$, whereas it decreased when the flankers were the digit $\langle 1\rangle$. Previous studies have suggested that other biases are likely to affect performance in this task (de Hevia et al. 2006; Fischer 2001). This should be kept in mind when interpreting the results obtained with neglect patients.

\section{Leftward bias following right neglect}

Patients with schizophrenia misbisect to the left of the veridical midpoint in line bisection tasks, revealing right minineglect as a consequence of a putative dysfunction of the left hemisphere (Michel et al. 2007). Cavézian et al. (2007) asked patients with schizophrenia to perform number interval bisection. If neglect direction were the critical factor determining the number interval bisection bias (see Zorzi et al. 2002), a leftward deviation as a function of number interval length should have been observed. Results showed that patients with schizophrenia misbisect to the left of the correct central number as a function of number interval length. Similar results have been reported in a single case with right neglect following damage to the left posterior superior parietal lobe (Pia et al. 2008). A right neglect patient misbisected to the left of the veridical midpoint both in visual line bisection and in number interval bisection. Thus, the direction of neglect (left vs. right) seems to be the crucial factor underlying the direction of bisection (rightward vs. leftward) as a function of stimulus length.

Numerical versus non-numerical ordered sequences

Zorzi et al. (2006) tested whether neglect affects, besides the MNL, also other non-numerical ordered sequences. Neglect patients and healthy controls performed visual line bisection and mental bisection of number (1-9), letter $(\mathrm{N}-\mathrm{V})$, and month (April-December) intervals. Results revealed a virtually identical pattern for visual lines and numbers, consisting in a rightward shift for longer lines/ number intervals and a leftward shift for the shortest line/ number interval (i.e., the crossover effect). Letter bisection revealed an overall rightward deviation that was not affected by interval length, suggesting a possible categorical representation for letters (left vs. right). There was no effect of neglect on months, although a trend towards a leftward deviation was suggestive of a circular (i.e., clock-like) representation. The authors concluded the spatial layout characterizing numerical representations constitutes a specific property of numbers rather than a general characteristic of ordered sequences. Zamarian et al. (2007) reported a rightward shift in mental letter bisection (i.e., left-to-right oriented line; for similar findings in neglect and pseudoneglect, see also Nicholls et al. 2008; Nicholls and Loftus 2007) and a leftward shift in mental day bisection (right-toleft circular representation). Finally, month bisection was not affected by neglect. Further research is required to shed light on the putative spatial nature of non-numerical ordinal sequences. It is also worth noting that, while numerical cues produced lateral shifts of spatial attention in the TOJ task used by Casarotti et al. (2007), letter cues did not yield any significant effect in otherwise identical experiments of the same study (see also Dehaene et al. 2003; Dodd et al. 2008; Fischer 2003, for the absence of SNARC-like effects with letters).

Neglect rehabilitation and number interval bisection

In a seminal study, Rossetti et al. (1998) showed that the performance of left neglect patients can be improved following a short adaptation period to prisms deviating the visual field $10^{\circ}$ to the right. Neglect patients were encouraged to perform a series of pointing movements that recalibrated the original deviation to the right into a more accurate straight ahead pointing. Such a visuomotor re-mapping allowed patients to explore the previously neglected space. In subsequent studies, prism adaptation was shown to ameliorate many aspects of left neglect, 
including line bisection (Farnè et al. 2002) and imaginal neglect (Rode et al. 2001).

If the MNL has a spatial nature, the number interval bisection task should not only be affected by spatial neglect but it should also be ameliorated by neglect rehabilitation. Rossetti et al. (2004) tested this hypothesis by asking two left neglect patients (FJY and GM) to bisect number intervals. Before prism adaptation, both patients showed a reliable shift of the observed midpoint to the right of the true midpoint. Then the two patients performed a short session of prism adaptation ( $8 \mathrm{~min}$ ). Following adaptation, they were asked to bisect again the mental number intervals that had been presented during the pre-adaptation session. Results showed a significant improvement in number interval bisection in both patients, following prism adaptation. Notably, the patient with the larger improvement in number interval bisection (FJY: 88\%) also showed the larger adaptation aftereffect, an index of prism efficacy. These findings further corroborated the intimate relation between space and numbers. Indeed, the reorganization of visuo-motor links subserved by a parietal-cerebellar circuit seems to affect the allocation of spatial attention along the MNL, as in the case of visual line bisection and of mental image inspection.

\section{Chronometric studies: reaction times and P300 latency}

Vuilleumier et al. (2004) tested left neglect patients performing number comparison. Patients were asked to decide whether a single Arabic digit presented at fixation was smaller (i.e., 1-4) or larger (i.e., 6-9) than 5. Results showed that the number on the immediate "left" (i.e., 4) of the reference number (i.e., 5) was responded to slower than the number on the immediate "right" (i.e., 6). In contrast, performance of healthy controls and of patients with right brain damaged but without left neglect was not affected by the position of the presented numbers along the MNL. In addition, when patients were asked to judge whether the same Arabic digits were smaller (1-6) than a new reference number (i.e., " 7 "), they became slower in processing the number to the immediate left of 7 , which was now number 6. Note that 6 was previously associated with the right, "good" space when the reference number was 5 . Thus, the same number can be processed faster or slower depending on whether it is on the relative right (5-6) or left (6-7) of a reference number. This response pattern could be explained by shifts of attention to the "left" and to the "right" of a specific number interval on the MNL. Thus, spatial attention resources are not distributed along the absolute left and right on the MNL but they are allocated according to the relative left and right defined by the reference number. This is in accordance with the original findings of Bisiach and Luzzatti (1978), who showed that the neglected side of a mental image depended on the subjective viewpoint of the patient: in effect, specific landmarks that had been omitted when they were on the left of a specific viewpoint were in contrast reported when patients inspected their mental image from the opposite viewpoint. Thus, allocation of spatial attention on the MNL or on other imaginal representations is affected by specific points of reference.

Imaginal neglect for the number space has been recently investigated in a psychophysiological study by using eventrelated potentials. Priftis et al. (2008) asked patients to pay attention to a rare spoken number word (1 or 9) and to ignore a frequent spoken number word (5). Results showed that, with respect to right brain damaged controls without neglect, left neglect patients had slower P300 brain waves to small numbers, whereas they had faster P300s to larger numbers. This pattern resembles that of attention allocation in neglect patients for the visual space, as reported by Làdavas et al. (1990). However, RTs to "one" were not slower than RTs to "nine". This finding is apparently at odds with that of Vuilleumier et al. (2004). Nonetheless, Vuilleumier et al. used a number comparison task, whereas Priftis et al. used an oddball task. Furthermore, Vuilleumier et al. reported slower RTs only for the numbers immediately to the left (e.g., 4) of the reference number (e.g., 5), whereas the target number in Priftis et al. was very distant from the non-target (i.e., the reference).

\section{Implicit versus explicit processing of the MNL}

One of the most intriguing phenomena in the neuropsychological literature of neglect is the dissociation between implicit and explicit processing of contralesional information (see Berti 2002, for review). Neglect patients demonstrate spared implicit processing of information that is ignored at the conscious (explicit) level. A patient (Marshall and Halligan 1988), for example, was presented with two vertically arranged black and white house drawings. The right halves of the house drawings were identical, whereas the left halves were not, due to the presence of depicted coloured flames in one house drawing. Although the patient claimed that the two houses were identical, presumably based on her explicit knowledge of the right half of the house drawings, she choose systematically the house drawing without flames as her preferred domicile. Thus, the patient's correct choice revealed intact contralesional implicit processing of information, which was ignored in the explicit judgment.

Similar dissociation between explicit and implicit processing were reported in various neglect studies. Berti and Rizzolatti (1992) showed that neglect patients were faster in categorising drawings presented in their intact hemispace when these drawings were accompanied by identical or semantically related drawings presented in the neglected 
hemispace. Thus, neglect patients could implicitly process contralesional spatial information. Similarly, Làdavas et al. (1993) described a neglect patient who was significantly facilitated at judging the lexical status (lexical decision) of letter strings presented in his ipsilesional intact hemispace when these strings were preceded by semantically related words, displayed briefly in his contralesional hemispace. It was shown that the patient lacked any conscious experience of the contralesional words.

Dissociations between implicit and explicit processing were reported in the domain of neglect dyslexia as well (Arduino et al. 2003; Làdavas et al. 1997; Vallar et al. 1996). In these studies, neglect patients produced paralexias affecting the contralesional portion of visually presented words. However, the same patients performed better in tasks requiring lexical decision or semantic categorisation of the same words that they could not read aloud.

In summary, the studies cited above suggest that neglect is a selective disorder of conscious processing of contralesional spatial information, whereas implicit processing of the same information might take place up to the semantic level. The ignored information can influence performance of neglect patients. Priftis et al. (2006) investigated whether the explicit versus implicit processing distinction applies to numerical processing too. They hypothesised that number interval bisection is a numerical task requiring the explicit processing of the MNL's spatial frame. Indeed, one is induced to activate and manipulate the MNL if making recourse to an algorithm (e.g., calculating the mean of the two numbers) is not allowed. For this reason, neglect can significantly affect this task (as reviewed above). On the other hand, the SNARC effect arises in tasks in which the task-irrelevant spatial frame of the MNL is activated automatically and implicitly (e.g., parity judgment: Dehaene et al. 1993; phoneme monitoring: Fias et al. 1996). Therefore, if the implicit versus explicit processing dissociation holds in the case of MNL processing, neglect should affect number interval bisection (i.e. the task requiring explicit processing of the MNL) in the presence of an intact SNARC effect (i.e. the task requiring implicit processing of the MNL).

Results confirmed the prediction. Neglect patients showed, again, a specific rightward bias in bisecting number intervals. Also, for the larger number intervals, there was a progressive shift to the right of the midpoint with increasing number interval length. In contrast, the midpoint was significantly shifted to the left for the shortest number interval (i.e., 3). Neglect patients, instead, showed a normal SNARC effect: They were faster in processing small number with the left effector and faster in processing larger numbers with the right effector. Priftis et al. argued that the intact SNARC effect in neglect patients reveals that the MNL is intact and that neglect affects allocation of spatial attention over an intact MNL. In conclusion, the dissociation between explicit and implicit processing in neglect can be observed also in the domain of the MNL.

\section{Pseudoneglect in number space}

Attentional asymmetries have been also reported in studies on visual line bisection with healthy participants. Bowers and Heilman (1980) were the first to show that neurologically intact participants misbisect to the left of the veridical midpoint. In their meta-analytic review of pseudoneglect, Jewell and McCourt (2000) showed that pseudoneglect is a systematic phenomenon that, nonetheless, can be modulated by a number of variables, such as age, sex, handedness, hand use, and direction of motor scanning.

Goebel et al. (2006) first explored the presence of pseudoneglect for the number space, by asking healthy participants to bisect number intervals. Results revealed that participants misbisected to the left of the midpoint number, as if they were allocating their spatial attention along a visual line (i.e. pseudoneglect). On some trials, number interval bisection took place following repetitive transcranial magnetic stimulation (rTMS) over the right parietal cortex, a brain region that is frequently damaged in case of neglect. Results showed that the leftward bias in number interval bisection was diminished following rTMS. The authors proposed that this could reflect a kind of neglect for the number space, because participants misbisected more to the right with respect to trials performed without rTMS. Nonetheless, a "true" neglect for the number space following rTMS would not cause misbisection to the right of the observed number but to the right of the veridical midpoint number. In contrast, what was really observed in the study of Goebel et al. was not a simulated neglect for the mental number line but an improvement in the participants' performance (i.e., less pseudoneglect).

The findings of Goebel et al. showed that pseudoneglect can be observed in the number space as it had been reported in the visual space. Visual line bisection was not tested in the study of Goebel et al., leaving the relation between visual line bisection and number interval bisection an open question. This issue was empirically explored in a study by Longo and Lourenco (2007). These authors tested whether pseudoneglect would affect number interval bisection in a way similar to that observed in visual line bisection. In their study, participants were asked to bisect not only visual lines, but also number intervals. Results showed that participants misbisected to the left of the veridical center (i.e., pseudoneglect) both in the visual line bisection task and in the mental number interval bisection task. Participants' performance in the two tasks was positively correlated, although pseudoneglect was stronger in the number interval bisection than in the visual line bisection task. Longo and 
Lourenco concluded that hemispheric asymmetries subserving spatial attention influence both visual and number space.

In one of the previous sections it was reported that adaptation to rightward deviating prisms improves neglect patients' performance not only on visual tasks but also in number interval bisection. Loftus et al. (2007) tested whether prism adaptation effects could be also observed in the case of pseudoneglect. First, healthy participants were shown triplets composed of three two-digit numbers (e.g., $16,36,55)$ and were required to judge whether the numerical distance was larger on the left (i.e., 16-36) or on the right (36-55) of the middle number. Participants overestimated the distance on the left of the middle number, confirming the presence of pseudoneglect for the mental number line (Goebel et al. 2006; Longo and Lourenco 2007). Second, participants underwent a brief period of adaptation to leftward deviating prisms that caused a rightward sensorimotor after-effect. Finally, participants performed again the triplet estimation task without wearing prisms. Results showed that prism adaptation corrected the leftward bias that was observed in the pre-adaptation phase. Thus, prism adaptation can recalibrate the leftward bias of number space in healthy participants and can diminish pseudoneglect for number space. Visual line bisection was not tested in the study of Loftus et al., and further studies on the effects of prism adaptation both on visual line bisection and on number interval bisection are required to examine the differential efficacy of prisms on these distinct spatial representations (visual space vs. number space).

In all the aforementioned studies, pseudoneglect was investigated using bisection (Goebel et al. 2006; Longo and Lourenco 2007) or similar numerical spatial tasks (Loftus et al. 2007). Loetscher and Brugger (2007) showed that pseudoneglect for the MNL is observed also in tasks that do not require number interval bisection. They re-analysed 16 studies in which the Mental Dice Task was employed. In this task, participants were required to randomly generate numbers by imagining 66 consecutive rolls of a die. Data reanalysis showed that participants significantly preferred to generate small numbers. These results are consistent with the presence of number pseudoneglect in random generation tasks too. ${ }^{2}$

In summary, pseudoneglect is present not only in visual line bisection but also in number interval bisection. How-

\footnotetext{
$\overline{{ }^{2} \text { Alternatively, }}$ one may think that smaller numbers are cognitively more available, perhaps because of their higher frequency. However, this interpretation is contradicted by the observation that the effect is modulated by head rotation (Loetscher et al. 2008): Participants showed a bias towards producing smaller numbers when their head (along with their spatial attention, presumably) was rotated to the left, whereas they produced larger numbers when their head was rotated to the right.
}

ever, Jewell and McCourt (2000) showed that pseudoneglect is influenced by different independent variables (i.e., age, sex, handedness, hand use, and direction of motor scanning). Thus, further research is required to explore the role of these variables on number interval bisection in healthy participants.

\section{Conclusion}

The available evidence is strongly in favor of an analogue, spatial representation of number magnitude, which is known as the mental number line (MNL). Its existence and characteristics are inferred by the finding that the time to compare two numbers in terms of magnitude decreases as a function of the numerical distance between them, that small digits prime left-side responses, whereas large digits prime right-side responses in a parity judgment task, and that small and large digits prime detection of left- and right-side targets, respectively. However, the most compelling evidence of the spatial nature of the representation of number magnitude comes from neuropsychological studies on neglect patients. In a task that requires bisecting a number interval, neglect patients systematically misplace the midpoint of the numerical interval to the right, in a way that closely resembles the typical pattern found in bisection of true visual lines, including the modulating effect of line length and the crossover effect with very short numerical intervals. These effects may be confined to numbers and do not seem to extend to items of ordered, non-numerical sequence. If the MNL has a spatial nature, the number interval bisection task should not only be affected by a spatial deficit like neglect. Performance in this task should also be ameliorated by procedures that are effective in rehabilitating neglect. That is exactly what results have shown. Converging evidence is provided by studies on pseudoneglect in healthy participants. Finally, the existence of a core spatial code for numbers fits well with the observation that numerical representations are deeply rooted in those cortical networks that also subserve spatial cognition (Hubbard et al. 2005; Walsh 2003).

Acknowledgments Preparation of this paper was supported by grants from MIUR (Prin 2005 and Prin 2006) to CU and MZ and from CARIPARO Foundation to MZ and KP.

\section{References}

Anderson B (1993) Spared awareness for the left side of internal visual images in patients with left-sided extrapersonal neglect. Neurology 1:213-216

Arduino LS, Burani C, Vallar G (2003) Reading aloud and lexical decision in neglect patients: a dissociation. Neuropsychologia 41:877-885 
Berti A (2002) Unconscious processing in neglect. In: Karnath HO, Milner AD, Vallar G (eds) The cognitive and neural bases of spatial neglect. Oxford University Press, Oxford, pp 313-326

Berti A, Rizzolatti G (1992) Visual processing without awareness: evidence from unilateral neglect. J Cogn Neurosci 4:345-351

Bisiach E, Luzzatti C (1978) Unilateral neglect of representational space. Cortex 14:129-133

Bisiach E, Rusconi ML, Peretti VA, Vallar G (1994) Challenging current accounts of unilateral neglect. Neuropsychologia 11:14311434

Bonato M, Priftis K, Marenzi R, Zorzi M (2008a) Modulation of hemispatial neglect by directional and numerical cues in the line bisection task. Neuropsychologia 46:423-433

Bonato M, Priftis K, Marenzi R, Zorzi M (2008b) Normal and impaired orienting of attention following central non-predictive cues. J Cogn Neurosci (in press)

Bowers D, Heilman KM (1980) Pseudoneglect: effects of hemispace on a tactile line bisection task. Neuropsychologia 18:491-498

Cappelletti M, Freeman ED, Cipolotti L (2007) The middle house or the middle floor: bisecting horizontal and vertical mental number lines in neglect. Neuropsychologia 45:2989-3000

Casarotti M, Michielin M, Zorzi M, Umiltà C (2007) Temporal order judgment reveals how number magnitude affects visuospatial attention. Cognition 102:101-117

Cavézian C, Rossetti Y, Danckert J, d'Amato T, Dalery J, Saoud M (2007) Exaggerated leftward bias in the mental number line of patients with schizophrenia. Brain Cogn 63:85-90

Chokron S, Bernard JM, Michel I (1997) Length representation in normal and neglect subjects with opposite reading habits studied through a line extension task. Cortex 35:47-64

Dehaene S (1992) Varieties of numerical abilities. Cognition 44:1-42

Dehaene S (2003) The neural basis of the Weber-Fechner law: a logarithmic mental number line. Trends Cogn Sci 7:145-147

Dehaene S, Dupoux E, Mehler J (1990) Is numerical comparison digital? Analogical and symbolic effects in two-digit number comparison. J Exp Psychol Hum Percept Perform 16:626-641

Dehaene S, Bossini S, Giraux P (1993) The mental representation of parity and number magnitude. J Exp Psychol Gen 122:371-396

Dehaene S, Piazza M, Pinel P, Cohen L (2003) Three parietal circuits for number processing. Cogn Neuropsychol 20:487-506

Dodd MD, Stigchel SV, Adil Leghari M, Fung G, Kingstone A (2008) Attentional SNARC: there's something special about numbers (let us count the ways). Cognition 108:810-818

Doricchi F, Guariglia P, Gasparini M, Tomaiuolo F (2005) Dissociation between physical and mental number line bisection in right hemisphere brain damage. Nat Neurosci 8:1663-1666

Farnè A, Rossetti Y, Toniolo S, Làdavas E (2002) Ameliorating neglect with prism adaptation: Visuo-manual and visuo-verbal measures. Neuropsychologia 40:718-729

Fias W, Brysbaert M, Geypens F, d'Ydewalle G (1996) The importance of magnitude information in numerical processing: Evidence from the SNARC effect. Mathem Cogn 2:95-110

Fischer MH (2001) Number processing induces spatial performance bias. Neurology 57:822-826

Fischer MH (2003) Spatial representations in number processing: evidence from a pointing task. Vis Cogn 10:493-508

Fischer MH, Castel AD, Dodd MD, Pratt J (2003) Perceiving numbers causes spatial shifts of attention. Nat Neurosci 6:555-556

Fischer MH, Warlop N, Hill RL, Fias W (2004) Oculomotor bias induced by number perception. Exp Psychol 51:91-97

Galfano G, Rusconi E, Umiltà C (2006) Number magnitude orients attention, but not against one's will. Psychon Bull Rev 13:869874

Gallistel CR, Gelman R (2000) Non-verbal numerical cognition: from reals to integers. Trends Cogn Sci 4:59-65

Galton F (1880) Visualised numerals. Nature 21:252-256
Gevers W, Ratinckx E, De Baene W, Fias W (2006a) Further evidence that the SNARC effect is processed along a dual-route architecture: evidence from the lateralized readiness potential. Exp Psychol 53:58-68

Gevers W, Verguts T, Reynvoet B, Caessens B, Fias W (2006b) Numbers and space: a computational model of the SNARC effect. J Exp Psychol Hum Percept Perform 32:32-34

Goebel S, Calabria M, Farnè A, Rossetti Y (2006) Parietal rTMS distorts the mental number line: simulating 'spatial' neglect in healthy subjects. Neuropsychologia 44:860-868

Guariglia C, Padovani AP, Pantano O, Pizzamiglio L (1993) Unilateral neglect restricted to visual imagery. Nature 364:235-237

Halligan PW, Marshall JC (1988) How long is a piece of string? A study of line bisection in a case of visual neglect. Cortex 24:321328

Halligan PW, Fink GR, Marshall JC, Vallar G (2003) Spatial cognition: evidence from visual neglect. Trends Cogn Sci 3:125-133

Heilman KM, Watson RT, Valenstein E (1979) Neglect and related disorders. In: Heilman KM, Valenstein E (eds) Clinical neuropsychology. Oxford University Press, New York, pp 268-307

de Hevia MD, Girelli L, Vallar G (2006) Number and space: a cognitive illusion? Exp Brain Res 168:254-264

Hoeckner SH, Zauner H, Moeller K, Wood G, Haider C, Gaßner A Nuerk HC (2008) Impairments of the mental number line for twodigit numbers in neglect. Cortex 44:429-438

Holyoak KJ (1977) The form of analog size information in memory. Cog Psych 9:31-51

Hubbard EM, Piazza M, Pinel P, Dehaene S (2005) Interactions between number and space in parietal cortex. Nat Rev Neurosci 6:435-448

Jewell G, McCourt ME (2000) Pseudoneglect: a review and meta-analysis of performance factors in line bisection tasks. Neuropsychologia 38:93-110

Keus IM, Schwarz W (2005) Searching for the functional locus of the SNARC effect: evidence for a response related origin. Mem Cognit 33:681-695

Keus IM, Jenks KM, Schwarz W (2005) Psychophysiological evidence that the SNARC effect has its functional locus in a response selection stage. Cogn Brain Res 24:48-56

Kosslyn SM, Murphy GL, Bamesderfer ME, Feinstein KJ (1977) Category and continuum in mental comparisons. J Exp Psych Gen 106:341-375

Làdavas E, Petronio A, Umiltà C (1990) The deployment of visual attention in the intact field of hemineglect patients. Cortex 26:307-317

Làdavas E, Paladini R, Cubelli R (1993) Implicit associative priming in a patient with left visual neglect. Neuropsychologia 12:1307-1320

Làdavas E, Umiltà C, Mapelli D (1997) Lexical and semantic processing in the absence of word reading: Evidence from neglect dyslexia. Neuropsychologia 35:1075-1085

Loetscher M, Brugger P (2007) Exploring number space by random digit generation. Exp Brain Res 180:655-665

Loetscher M, Schwarz U, Schubiger M, Brugger P (2008) Head turns bias the brain's internal random generator. Curr Biol 18:R60-R62

Loftus AM, Nicholls ME, Mattingley JB, Bradshaw JL (2007) Left to right: representational biases for numbers and the effect of visuomotor adaptation. Cognition 107:1048-1058

Loftus AM, Nicholls ME, Mattingley JB, Chapman HL, Bradshaw JL (2008) Pseudoneglect for the bisection of mental number lines. Q J Exp Psychol (in press)

Longo MR, Lourenco SF (2007) Spatial attention and the mental number line: evidence for characteristic biases and compression. Neuropsychologia 45:1400-1407

Lu CH, Proctor RW (1995) The influence of irrelevant location information on performance-a review of the Simon and spatial Stroop effects. Psychon Bull Rev 2:174-207 
Marshall JC, Halligan PW (1988) Blindsight and insight in visuospatial neglect. Nature 336:766-767

Marshall JC, Halligan PW (1989) When right goes left: an investigation of line bisection in a case of visual neglect. Cortex 25:503-515

Michel C, Cavezian C, d'Amato T, Dalery J, Rode G, Saoud M, Rossetti Y (2007) Pseudoneglect in schizophrenia: a line bisection study with cueing. Cognit Neuropsychiatry 12:222-234

Moyer RS, Landauer TK (1967) The time required for judgements of numerical inequality. Nature 215:1519-1520

Nicholls ME, Loftus AM (2007) Pseudoneglect and neglect for mental alphabet lines. Brain Res 1152:130-138

Nicholls ME, Kamer A, Loftus AM (2008) Pseudoneglect for mental alphabet lines is affected by prismatic adaptation. Exp Brain Res 191:109-115

Pia L, Corazzino LL, Folegatti A, Gindri P, Cauda F (2008) Mental number line disruption in a right-neglect patient after left-hemisphere stroke. Brain Cogn (in press)

Priftis K, Zorzi M, Meneghello F, Marenzi R, Umiltà C (2006) Explicit versus implicit processing of representational space in neglect: dissociations in accessing the mental number line. J Cogn Neurosci 18:680-688

Priftis K, Piccione F, Giorgi F, Meneghello F, Umiltà C, Zorzi M (2008) Lost in number space after right brain damage: a neural signature of representational neglect. Cortex 44:449-453

Proctor RW, Cho YS (2006) Polarity correspondence: a general principle for performance of speeded binary classification tasks. Psychol Bull 132:416-442

Restle F (1970) Speed of adding and comparing numbers. J Exp Psych 83:274-278

Ristic J, Wright A, Kingstone A (2006) The number line effect reflects top-down control. Psychon Bull Rev 113:862-868

Rode G, Rossetti Y, Boisson D (2001) Prism adaptation improves representational neglect. Neuropsychologia 39:1250-1254

Rossetti Y, Rode G, Pisella L, Farnè A, Li L, Boisson D, Perenin MT (1998) Prism adaptation to rightward optical deviation rehabilitates left hemispatial neglect. Nature 395:166-169
Rossetti Y, Jacquin-Courtois S, Rode G, Ota H, Michel C, Boisson D (2004) Does action make the link between number and space representation? Psychol Sci 15:426-430

Santens S, Gevers W (2008) The SNARC effect does not imply a mental number line. Cognition 108:263-270

Schwarz W, Keus IM (2004) Moving the eyes along the mental number line: comparing SNARC effects with saccadic and manual responses. Percept Psychophys 66:651-664

Schwarz W, Müller D (2006) Spatial associatiosn in number-related tasks: a comparison of manual and pedal responses. Exp Psychol 53:4-15

Stoianov I, Kramer P, Umiltà C, Zorzi M (2008) Visuospatial priming of the mental number line. Cognition 106:770-779

Vallar G, Guariglia C, Nico D, Tabossi P (1996) Left neglect dyslexia and the processing of neglected information. J Clin Exp Neuropsychol 18:733-746

Vuilleumier P, Ortigue S, Brugger P (2004) The number space and neglect. Cortex 40:399-410

Walsh V (2003) A theory of magnitude: common cortical metrics of time, space, and quantity. Trends Cogn Sci 7:483-488

Woocher FD, Glass AL, Holyoak KJ (1978) Positional discriminability in linear orderings. Mem Cognit 6:165-173

Zamarian L, Egger C, Delazer M (2007) The mental representation of ordered sequences in visual neglect. Cortex 43:542-550

Zorzi M, Umiltà C (1995) A computational model of the Simon effect. Psychol Res 58:193-205

Zorzi M, Priftis K, Umiltà C (2002) Neglect disrupts the mental number line. Nature 417:138-139

Zorzi M, Priftis K, Meneghello F, Marenzi R, Umiltà C (2004) Dissociations in the bisection of visual lines and number lines in neglect. Poster presented at the first congress of the European Neuropsychological Societies, Modena, Italy

Zorzi M, Priftis K, Meneghello F, Marenzi R, Umiltà C (2006) The spatial representation of numerical and non-numerical sequences: evidence from neglect. Neuropsychologia 44:1061-1067 